\title{
A CHARACTERIZATION OF THE MULTIPARAMETER WIENER PROCESS AND AN APPLICATION
}

\author{
CHANG C. Y. DOREA
}

\begin{abstract}
In this note we give an application of a characterization of the multiparameter Wiener process and of the invariance principle for $R^{k}$-valued martingales.
\end{abstract}

1. Introduction. Let $q$ be a positive integer, $I^{q}=[0,1]^{q}$, the points in $I^{q}$ will be denoted by $\mathbf{t}, \mathbf{s},\left(t_{1}, t_{2}, \ldots, t_{q}\right)$, etc. $I^{q}$ is partially ordered by stipulating $\mathbf{t} \leq \mathbf{s}$ if and only if $t_{i} \leq s_{i}$ for $i=1, \ldots, q$. A block $B$ in $I^{q}$ is a subset of the form $B=(\mathrm{s}, \mathrm{t}]=\prod_{j=1}^{q}\left(s_{j}, t_{j}\right)$ for $0 \leq \mathrm{s} \leq \mathrm{t} \leq 1$. For a function $f$ defined on $I^{q}$ we define the increment of $f$ around the block $B$ by

$$
f(B)=\sum_{\substack{\epsilon_{j}=0,1 \\ j=1, \ldots, q}}(-1)^{q-\Sigma \epsilon_{j}} f\left(s_{1}+\epsilon_{1}\left(t_{1}-s_{1}\right), \ldots, s_{q}+\epsilon_{q}\left(t_{q}-s_{q}\right)\right) .
$$

Similarly if $C=(\mathbf{u}, \mathbf{v}]$ is a block of $I^{q-1}$ we define for $i=1, \ldots, q$ and $0 \leq$ $t \leq 1$

$$
\begin{aligned}
f^{(i)}(t ; C)= & \sum_{\substack{\epsilon_{j}=0,1 \\
j=1, \ldots, q-1}}(-1)^{q-1-\Sigma \epsilon_{j}} \\
& \cdot f\left(u_{1}+\epsilon_{1}\left(v_{1}-u_{1}\right), \ldots, u_{i-1}+\epsilon_{i-1}\left(v_{i-1}-u^{i-1}\right),\right. \\
& \left.\quad t, u_{i}+\epsilon_{i}\left(v_{i}-u_{i}\right), \ldots, u_{q-1}+\epsilon_{q-1}\left(v_{q-1}-u_{q-1}\right)\right) .
\end{aligned}
$$

Notice

$$
f^{(i)}(t ; C)=f\left(\left(\left(u_{1}, \ldots, u_{i-1}, 0, u_{i}, \ldots, u_{q-1}\right),\left(v_{i}, \ldots, v_{i-1}, t, v_{i}, \ldots, v_{q-1}\right)\right)\right)
$$

if $f(\mathrm{~s})=0$ whenever $s_{i}=0$.

Let $C\left(I^{q}\right)$ be the set of all continuous functions on $I^{q}$ with the uniform metric and let $D^{q}=D\left(I^{q}\right)$ be the Skorohod function space on $I^{q}$. See Bickel-Wichura [1] for further details.

The Wiener process $\left\{W(\mathbf{t}), \mathbf{t} \in I^{q}\right\}$ on $D^{q}$ is characterized by

$$
\text { (a) } P\left(W \in C\left(I^{q}\right)\right)=1 \text {, }
$$

(b) $W(\mathbf{t})$ vanishes at the lower boundary of $I^{q}$,

that is, $P(W(\mathbf{t})=0)=1$ if $t_{i}=0$ for some $i$.

(c) If $B_{1}, B_{2}, \ldots, B_{k}$ are pairwise disjoint blocks of $I^{q}$ then the increments $W\left(B_{1}\right), \ldots, W\left(B_{k}\right)$ are independent normal random variables with mean zero and variances $\lambda_{q}\left(B_{1}\right), \ldots, \lambda_{q}\left(B_{1}\right), \lambda_{q}$ being the Lebesgue measure on $I^{q}$.

Lemma 1 below gives a characterization of the $q$-parameter Wiener process in terms of $k$-dimensional Wiener processes. Since its proof is straightforward it will be omitted.

Received by the editors June 22, 1981.

1980 Mathematics Subject Classification. Primary 60G60, 60J65; Secondary 60F17. 
LEMMA 1. Let $\left\{X(\mathrm{t}), \mathrm{t} \in I^{q}\right\}$ be a process that vanishes at the lower boundary of $I^{q}$ and has sample paths in $D^{q}$ with $P\left(X \in C^{q}\right)=1$. Then $X(t)$ is the Wiener process on $D^{q}$ if and only if for some $i(i=1, \ldots, q)$ the process $\left(X^{(i)}\left(t ; C_{1}\right), \ldots, X^{(i)}\left(t ; C_{k}\right)\right)$ is a $k$-dimensional Wiener process with the appropriate variance, where $k$ is an arbitrary positive integer and the $C_{j}$ 's are arbitrary disjoint blocks of $I^{q-1}$.

2. A convergence theorem. Let $(\Omega, \mathcal{F}, P)$ be a probability space and let $\left\{\mathcal{F}_{\mathbf{t}}, \mathrm{t} \in\right.$ $\left.I^{q}\right\}$ be a family of $\sigma$-subfields such that $\mathcal{F}_{\mathrm{a}} \subset \mathcal{F}_{\mathrm{t}}$ if $\mathrm{s} \leq \mathrm{t}$. For $0 \leq t \leq 1$ we define for $i=1, \ldots, q$

$$
\mathcal{F}_{t}^{(i)}=\mathcal{F}_{(1, \ldots, t, 1, \ldots, 1)} \text { with } t \text { on the } i \text { th position. }
$$

We shall consider random fields $\left\{X(t), t \in I^{q}\right\}$ adapted to a nondecreasing family of $\sigma$-fields $\left\{\mathcal{F}_{t}^{X}\right\}$ that vanishes at the lower boundaries of $I^{q}$. By taking $\mathcal{F}_{t}=\mathcal{F}_{t}^{X} X$ $F_{\mathrm{t}}$ for $\mathrm{t} \in I^{q}$ we may consider $X(\mathrm{t})$ and $W(\mathrm{t})$ adapted to the same family of $\sigma$-fields $\left\{f_{t}\right\}$, where $F_{t}=\sigma(W(\mathrm{~s}), \mathrm{s} \leq \mathrm{t})$.

THEOREM 1. Let $\left\{X_{n}(t), t \in I^{q}\right\}_{n \geq 1}$ be a sequence of processes adapted to $\mathcal{F}_{t}$ with sample paths in $D^{q}$. Fix $i$. Suppose that for $k=1,2, \ldots$ and $C_{1}, \ldots, C_{k}$ disjoint blocks of $I^{q-1}$ we have

$$
\left(X_{n}^{(i)}\left(s^{1} ; C_{1}\right), \ldots, X_{n}^{(i)}\left(s^{k} ; C_{k}\right)\right) \stackrel{D}{\rightarrow}\left(W^{(i)}\left(s^{1} ; C_{1}\right), \ldots, W^{(i)}\left(s^{k} ; C_{k}\right)\right)
$$

for $s^{1}, s^{2}, \ldots, s^{k} \in I$, where $\stackrel{D}{\rightarrow}$ stands for convergence in distribution.

Then the finite dimensional distributions of $X_{n}$ converge to those of $W$.

Proof. (i) For $\mathrm{t} \in I^{q}$ we have $X_{n}(\mathrm{t}) \stackrel{D}{\rightarrow} W(\mathrm{t})$ since $X_{n}(\mathrm{t})=X_{n}^{(i)}\left(t_{i} ; C\right)$ where $C=\prod_{j \neq i}\left(0, t_{j}\right]$.

(ii) To see that $\left(X_{n}\left(\mathbf{t}^{1}\right), \ldots, X_{n}\left(\mathbf{t}^{k}\right)\right) \stackrel{D}{\rightarrow}\left(W\left(\mathbf{t}^{1}\right), \ldots, W\left(\mathbf{t}^{k}\right)\right)$ for $\mathbf{t}^{1}, \ldots, \mathbf{t}^{k} \in I^{q}$, write $X_{n}\left(t^{l}\right)=X_{n}^{(i)}\left(t_{i}^{l} ; C_{l}\right)$ and $W\left(t^{l}\right)=W^{(i)}\left(t_{i}^{l} ; C_{l}\right)$ with $C_{l}=\prod_{j \neq i}\left(0, t_{j}^{l}\right]$. The $I^{q-1}$ blocks $C_{1}, \ldots, C_{k}$ are not necessarily disjoint, but given $a_{1}, a_{2}, \ldots, a_{k} \in R$ we can write $\sum_{l=1}^{k} a_{l} X_{n}^{(i)}\left(t_{i}^{l} ; C_{l}\right)$ as a linear combination of random variables of the form $X_{n}^{(i)}\left(t_{i}^{p} ; E_{p}\right)$ where the $E_{p}$ 's are disjoint blocks of $I^{q-1}$.

3. Invariance principle for $R^{k}$-valued martingale. Theorem 2 below is just a $k$-dimensional version of Billingsley's theorem [2]. Although we could prove it by using either Billingsley's arguments or McLeish's arguments [8], it can be done by Trotter's operator method [10] as outlined below.

THEOREM 2. Let $Y_{1}, Y_{2}, \ldots$ be a sequence of $R^{k}$-valued random vectors, $Y_{n}=$ $\left(Y_{n}(1), \ldots, Y_{n}(k)\right)$, stationary, ergodic and such that

$$
\begin{aligned}
& E\left(Y_{n}^{2}(j)\right)=\alpha_{j}, \quad \text { positive and finite, } \\
& E\left(Y_{n}(i) Y_{n}(j)\right)=0, \quad i \neq j, \\
& E\left(Y_{n} \mid Y_{1}, \ldots, Y_{n-1}\right)=0 .
\end{aligned}
$$

Then $S_{n}=\sum_{i=1}^{n} Y_{i} / \sqrt{n}$ converges in distribution to $Z$, where $Z$ has the multivariate normal distribution with mean zero, $E Z^{2}(j)=\alpha_{j}$ and $E Z(i) Z(j)=0$ for $i \neq j$. 
ProOF. To avoid messy notation let $k=2$. For a random vector $(V, U)$ define

$$
T_{V, U} f(x) g(y)=\iint f(v+x) g(u+y) d F_{V, U}(x, y) .
$$

Let $C_{0}^{2}=\left\{f: R \rightarrow R\right.$ such that $f, f^{\prime}, f^{\prime \prime}$ are bounded and uniformly continuous $\}$. To show that $\left(S_{n}(1), S_{n}(2)\right) \stackrel{D}{\rightarrow}(Z(1), Z(2))$ it is enough to verify that for all $f, g \in C_{0}^{2}$ we have

$$
\lim _{n \rightarrow \infty}\left|T_{S_{n}(1), S_{n}(2)} f g-T_{Z(1), Z(2)} f g\right|=0 .
$$

Write

$$
\left(T_{S_{n}}-T_{Z}\right) f g=(A+B) f g
$$

where

$$
B=T_{U_{1}} \cdots T_{U_{n}}-T_{Z}
$$

and

$$
A=\sum_{j=0}^{n-2} T_{U_{n-j+1}} \cdots T_{U_{n+1}}\left[T_{V_{n-j}}-T_{U_{n-j}} T_{V_{n-j-1}}\right]
$$

with $U_{j}(i)=Y_{j}(i) / \sqrt{n}, U_{n+1}=(0,0)$ and $V_{j}(i)=\sum_{l=1}^{j} U_{l}(i)$. Since

$$
\begin{aligned}
T_{V_{n-j}} f(x) g(y)= & \int f\left(a_{1}+\cdots+a_{n-j}+x\right) \\
& \cdot g\left(b_{1}+\cdots+b_{n-j}+y\right) d F_{U_{1}, \ldots, U_{n-j}}\left(a_{1}, b_{1}, \ldots, a_{n-j}, b_{n-j}\right)
\end{aligned}
$$

we expand $f(x) g(y)$ in Taylor series around $\left(a_{1}+\cdots+a_{n-j-1}+x, b_{1}+\cdots+\right.$ $\left.b_{n-j-1}+y\right)$ and

$$
\begin{aligned}
T_{V_{n-j}} f(x) g(y)=E & \left(f(\cdot) g(\cdot)+U_{n-j}(1) f^{\prime}(\cdot) g(\cdot)\right. \\
& +U_{n-j}(2) f(\cdot) g^{\prime}(\cdot)+U_{n-j}(1) U_{n-j}(2) f^{\prime}(\cdot) g^{\prime}(\cdot) \\
& \left.+\frac{1}{2} U_{n-j}^{2}(1) f^{\prime \prime}(\cdot) g(\cdot)+\frac{1}{2} U_{n-j}^{2}(2) f(\cdot) g^{\prime \prime}(\cdot)+R_{j}\right)
\end{aligned}
$$

where $f(\cdot)=f\left(V_{n-j}(1)+x\right)$ and $g(\cdot)=g\left(V_{n-j}(2)+y\right)$. The term $R_{j}$ can be estimated as in [10], hence given $\epsilon>0$ for $n$ sufficiently large we have $E\left|R_{j}\right| \leq$ $\epsilon n^{-1}$. Making a similar estimate for $T_{U_{n-j}} T_{V_{n-j-1}}$ and using condition (3.1) we have

$$
\begin{aligned}
\left(T_{V_{n-j}}-T_{U_{n-j}} T_{V_{n-j-1}}\right) f g & \\
=E\left(R_{j}+R_{j}^{\prime}+\frac{1}{2} f^{\prime \prime} g[\right. & \left.E\left(U_{n-j}^{2}(1) \mid G_{n-j-1}\right)-\frac{\alpha_{1}}{n}\right] \\
& \left.+\frac{1}{2} f g^{\prime \prime}\left[E\left(U_{n-j}^{2}(2) \mid G_{n-j-1}\right)-\frac{\alpha_{2}}{n}\right]\right)
\end{aligned}
$$

where $G_{j}=\sigma\left(Y_{1}, \ldots, Y_{j}\right)$.

With similar estimates for all the remaining terms we have

$$
\begin{aligned}
\left|\left(T_{S_{n}}-T_{Z}\right) f g\right| \leq & 2 \epsilon+\frac{1}{2}\left|f^{\prime \prime}\right||g| E\left(\left|\sum_{i=1}^{n} U_{i}^{2}(1)-\alpha_{1}\right|\right) \\
& +\frac{1}{2}|f|\left|g^{\prime \prime}\right| E\left(\left|\sum_{i=1}^{n} U_{i}^{2}(2)-\alpha_{2}\right|\right)
\end{aligned}
$$

where $|\cdot|$ is the supremum norm. The proof is completed by using the ergodicity. 
4. Application. In this section we shall consider a random net $\left\{\xi_{\mathbf{n}}, \mathbf{n} \in Z^{2}\right\}$, stationary with $E \xi_{\mathrm{n}}^{2}=\sigma^{2}>0$ and finite, where $Z$ stands for the integers. Let

$$
\begin{aligned}
& \mathcal{F}_{\mathbf{m}}=\sigma\left(\xi_{\mathbf{j}}, \mathbf{j} \leq \mathbf{m}\right), \quad \mathcal{F}_{\mathbf{t}}=\prod_{\mathbf{m}} \mathcal{F}\left(\left[m_{1} t_{1}\right],\left[m_{2} t_{2}\right]\right), \\
& S_{n_{1} 0}=S_{0 n_{2}}=0, \quad n_{1} \geq 0, n_{2} \geq 0 \text {, } \\
& S_{n_{1} 0}=S_{0 n_{2}}=0, \quad n_{1} \geq 0, n_{2} \geq 0 \text {, } \\
& S_{\mathbf{n}}=\sum_{\mathbf{1} \leq \mathbf{j} \leq \mathbf{n}} \xi_{\mathbf{j}}, \quad \mathbf{n} \geq \mathbf{1}, \\
& X_{\mathbf{n}}(\mathbf{t})=\left(\sigma^{2} n_{1} n_{2}\right)^{-1 / 2} S_{[\mathbf{n t}]}, \quad \mathbf{n} \geq \mathbf{1} \text { and } \mathbf{t} \in I^{2},
\end{aligned}
$$

where $[\mathbf{n t}]=\left(\left[n_{1} t_{1}\right],\left[n_{2} t_{2}\right]\right)$.

THEOREM 3. Let $\left\{\xi_{\mathbf{n}}, \mathbf{n} \in Z^{2}\right\}$ be a stationary and ergodic random net with $E \xi_{\mathbf{n}}^{2}=1$. Suppose further that $X_{\mathbf{n}}(\mathbf{t})$ is a strong martingale relative to $\boldsymbol{F}_{\mathbf{t}}$. Then $\left\{X_{\mathbf{n}}, \mathbf{n} \geq 1\right\}$ converges weakly, in $D^{2}$, to the two-parameter Wiener process.

REMARKS. (i) There is no loss of generality by considering $\sigma^{2}=1$ in our theorem.

(ii) For the concept of strong martingale see Cairoli-Walsh [4].

(iii) Since $X_{\mathbf{n}}$ is a strong martingale we have $E \xi_{\mathbf{n}}=0 \forall \mathbf{n}$ and $E\left(\xi_{\mathbf{n}} \mid \mathcal{F}_{m_{1}}^{(1)} \vee \mathcal{F}_{m_{2}}^{(2)}\right)=$ 0 if $n_{1}>m_{1}$ and $n_{2}>m_{2}$.

Proof. (a) Convergence of finite dimensional distributions. By Theorem 1 it is enough to show that

$$
\left(X_{\mathbf{n}}^{(1)}\left(s^{1} ; I_{1}\right), \ldots, X_{\mathbf{n}}^{(1)}\left(s^{k} ; I_{k}\right)\right) \stackrel{D}{\rightarrow}\left(W^{(1)}\left(s^{1} ; I_{1}\right), \ldots, W^{(1)}\left(s^{k} ; I_{k}\right)\right)
$$

for arbitrary $s^{1}, \ldots, s^{k}$ and disjoint intervals $I_{1}, \ldots, I_{k}$. But (4.1) is implied by Theorem 2 since $\left(X_{\mathbf{n}}^{(1)}\left(s^{1} ; I_{1}\right), \ldots, X_{\mathbf{n}}^{(1)}\left(s^{k} ; I_{k}\right)\right)$ is a $R^{k}$-valued martingale.

(b) To prove tightness we can follow Billingsley's arguments [3, pp. 207-208] and observing that

(b1) if $\mathbf{i} \neq \mathbf{j}$, say $i_{1}>j_{1}$, then

$$
E\left\{\xi_{\mathbf{i}} \xi_{\mathbf{j}}\right\}=E\left\{E\left(\xi_{\mathbf{i}} \xi_{\mathbf{j}} \mid \mathcal{F}_{j_{1}}^{(1)} \vee \mathcal{F}_{\min \left(i_{2}, j_{2}\right)}^{(2)}\right)\right\}=0
$$

so that $E S_{n}^{2}=n_{1} n_{2}$.

(b2) if $\left|\xi_{0}\right| \leq M<\infty$ with probability 1 then there exists a constant $\alpha$ such that

$$
E S_{\mathrm{n}}^{4} \leq \alpha n_{1}^{2} n_{2}^{2} M^{4}
$$

Notice the summands of $S_{\mathbf{n}}^{4}$ will be of the form $\xi_{\mathbf{i}} \xi_{\mathbf{j}} \xi_{\mathbf{k}} \xi_{\mathbf{l}}$.

Case 1. If $i_{1}>j_{1} \geq k_{1} \geq l_{1}$

$$
E\left(\xi_{\mathbf{i}} \xi_{\mathbf{j}} \xi_{\mathbf{k}} \xi_{\mathbf{l}}\right)=E\left(\xi_{\mathbf{j}} \xi_{\mathbf{k}} \xi_{\mathbf{l}} E\left(\xi_{\mathbf{i}} \mid \mathcal{F}_{j_{1}}^{(1)}\right)\right)=0 .
$$

Case 2. If $i_{1}=j_{1}>k_{1} \geq l_{1}$

$$
E\left(\xi_{\mathbf{i}} \xi_{\mathbf{j}} \xi_{\mathbf{k}} \xi_{\mathbf{l}}\right)\left\{\begin{array}{l}
=E\left(\xi_{\mathbf{j}} \xi_{\mathbf{k}} \xi_{\mathbf{l}} E\left(\xi_{i} \mid \mathcal{F}_{k_{2}}^{(1)} \vee \mathcal{F}_{j_{2}}^{(2)}\right)\right)=0, \quad i_{2}>j_{2}, \\
\leq M^{2}\left|E\left(\xi_{\mathbf{k}} \xi_{\mathbf{l}}\right)\right|=0 \quad \text { if } i_{2}=j_{2} \text { and } \mathbf{k} \neq 1, \\
\leq M^{4} \quad \text { if } i_{2}=j_{2} \text { and } \mathbf{k}=1,
\end{array}\right.
$$

similarly one can estimate all the remaining cases and get the estimate (4.2). 
(b3) $E\left(\max _{\mathbf{k} \leq \mathbf{n}}\left|S_{\mathbf{k}}\right|^{4}\right) \leq(4 / 3)^{8} E\left|S_{\mathbf{n}}\right|^{4}$.

This follows from Shorack-Smythe [9, Lemma 1] (cf. equation (4)).

(b4) If $\left|\xi_{0}\right| \leq M<\infty$ then

$$
E\left\{\max _{\mathbf{k} \leq \mathbf{n}}\left|S_{\mathbf{k}}\right|^{4}\right\} \leq \alpha^{\prime} n_{1}^{2} n_{2}^{2} M^{4} .
$$

(b5) Using Billingsley's truncation arguments the proof follows.

\section{REFERENCES}

1. P. J. Bickel and M. J. Wichura, Convergence criteria for multiparameter processes and some applications, Ann. Math. Statist. 42 (1971), 1656-1670.

2. P. Billingsley, The Lindeberg-Levy theorem for martingales, Proc. Amer. Math. Soc. 12 (1961), 788-792.

3. _ Convergence of probability measures, Wiley, New York, 1968.

4. R. Cairoli and J. B. Walsh, Stochastic integrals in the plane, Acta Math. 134 (1975), 111-183.

5. C. M. Deo, A functional central limit theorem for stationary random fields, Ann. Probab. 3 (1975), 708-715.

6. E. B. Dynkin, Markov processes, Springer-Verlag, Berlin, 1965.

7. T. G. Kurtz, Semigroups of conditioned shifts and approximation of Markov processes, Ann. Probab. 3 (1975), 618-642.

8. D. L. McLeish, Dependent central limit theorems and invariance principles, Ann. Probab. 2 (1974), 620-628.

9. G. R. Shorack and R. T. Smythe, Inequalities for $\max \left|S_{\mathbf{k}}\right| / b_{\mathbf{k}}$ where $\mathbf{k} \in N^{r}$, Proc. Amer. Math. Soc. 54 (1976), 331-336.

10. H. F. Trotter, An elementary proof of the central limit theorem, Arch. Math. 10 (1959), 225-234.

Departamento de MATEMÁtica-IE, Universidade de Brasília, 70.910 Brasfllia, DF, BRASIL 